\title{
Palliative care: a look at the experience in Southern Brazil
}

Keywords: chronic, alzheimer's diseases, agencies, famil, hospitalizations, skin, palliative care

Abbreviations: PIDI, programa de internação domiciliar interdisciplinar oncológico

\section{Introduction}

Population aging is accompanied by a high incidence of chronic degenerative diseases, such as Alzheimer's disease, cancer, diabetes mellitus, among others, drawing, on a world stage, the need for an expanded view of government agencies, in order to elabore effective public health policies in the care of people. In Brazil, both policies and health services for progressive diseases are still recent. The first services were organized in the 1990s in the state of São Paulo, and in 2011 the Ministry of Health launched the Best at Home Program (Programa Melhor em Casa). ${ }^{1}$

In this program of the country's government, municipalities can join. It is a home care service for people with temporary conditions, such as skin lesions, or definitive, referring to the terminal or even incurable diseases, aimed at care closer to the family, as well as stimulate the reduction of the number of hospitalizations and risks of hospital infections. It is in this context that palliative care is inserted in the country, with the purpose of providing quality of life for the families and relief of symptoms resulting from the disease, from multi professional perspective.

Thus, palliative care refers to patient and family-centered care that optimizes quality of life by anticipating, preventing, and treating suffering. Palliative care throughout the continuum of illness involves addressing physical, intellectual, emotional, social, and spiritual needs and facilitating patient autonomy, access to information, and choice..$^{2,3}$ In the palliative method the treatment will be successful if the person happens to live well despite the illness, even if he dies at any moment.

A number of challenges permeate the palliative care arena. One, of them, refers to the palliative team itself. In the experience of an Oncological Interdisciplinary Home Hospitalization Program (Programa de Internação Domiciliar Interdisciplinar OncológicoPIDI), in Hospital Escola of Federal University of Pelotas, the state of Rio Grande do Sul, gaps were identified in curricular training of professionals, to deal with death, palliative care; and teamwork. In order to overcome the inadequacies of vocational training, the workers sought theoretical subsidies in postgraduate courses, with the organization of annual events, insertion of academics and multi professional residents in the service. ${ }^{3}$ However, there was a climate of dialogue, respect, and exchange of affections among the multi professional team, to deal with the suffering coming from caring, understanding the death or its imminence as a natural process and assist in the morning of relatives.

Other challenges are also related to the difficulties of support early palliative care for patients with advanced cancer ${ }^{4}$ and of
Volume I Issue 3 - 2017

Adrize Rutz Porto, Julieta Maria Carriconde
Fripp, Isabel Cristina de Oliveira Arrieira
Department of Nursing, Federal University of Pelotas, Brazil

Correspondence: Adrize Rutz Porto, Department of Nursing, Federal University of Pelotas, Brazil,

Email adrizeporto@gmail.com

Received: March 31, 2017| Published: August 23, 2017

preparing the team to work with analgesia, to offer, in the same way, non-pharmacological treatment, with the purpose of extended clinical practice, of the integral care of both patients, their families, and caregivers, in the physical, psychic, social and spiritual aspects. Therefore, palliative care involves multiple dimensions of health care, making it complex. Similarly, when considering home care services, the eligibility criteria for admission of patients, such as the need for a caregiver that restricts access to this mode of care.

The PIDI team, which has been operating since 2005, provides home-based palliative care twice a day. The team of reference formed by a doctor, nurse, two nursing technicians, also counts on a support team consisting of nutritionist, medical coordinator of the team, surgeon physician, psychologist, physiotherapist, social worker and theologian, administrative assistant, driver. ${ }^{5}$ Still, it presents a group for the caregivers of the patients and, another, for mourners. In 2011, PIDI expanded to two teams, which serve about 250 patients annually, as well as having three reference teams of the Best in Home Program and one of support team providing approximately 1,500 patients in home care.

A research showed the profile of the 213 patients of the PIDI from 2005 to 2008 . There were metastases in $92 \%$ of the patients, of which $32 \%$ were not hospitalized during the period. Main reasons for hospitalization were anorexia, pain and asthenia; half of the patients evolved to death at home. ${ }^{6}$

Finally, despite advances, it is understood that, in Brazil, there is still much to be done in relation to the supply and organization of palliative care services. At the same time, there is an increasing incorporation of protocols in services to guide the practice of palliative care, systematization of health records. However, there is a need for a more efficient and effective health care network for giving an account of the demands to promote the autonomy of people facing chronicdegenerative diseases, as well as the quality of life and death.

\section{Acknowledgements}

None.

\section{Conflict of interest}

The author declares no conflict of interest. 


\section{References}

1. Brasil BF. Caderno de atenção domiciliar. Ministério da Saúde. 2012;2:2-207.

2. National quality forum. A national framework and preferred practices for palliative and hospice care quality. National Quality Forum, Washington, USA: Springer; 2006. p. 1-124.

3. Adrize Rutz Porto, Maira Buss Thofehrn, Daiane Dal Pai, et al. Vision of professionals about your work in interdisciplinary home internation program of cancer: a Brazilian reality. Av enferm. 2014;32(1):72-79.
4. Zimmermann C, Swami N, Krzyzanowska M, et al. Early palliative care for patients with advanced cancer: a cluster-randomised controlled trial. Lancet. 2014;383(9930):1721-1730.

5. Daniela Habekost Cardoso, Luana Amaral Mortola, Isabel Cristina de Oliveira Arrieira. Terapia subcutânea para pacientes em cuidados paliativos: a experiência de enfermeiras na atenção domiciliar. Journal of Nursing and Health. 2016;6(2):346-354.

6. Fripp JC, Facchini LA, Silva SM. Characterizing a home and palliative care program in the Municipality of Pelotas, State of Rio Grande do Sul, Brazil: a contribution to full attention to cancer patients at the National Unified Health System. Epidemiol Serv Saúde. 2012;21(1):69-78. 